\title{
Numerical Solutions for the Time and Space Fractional Nonlinear Partial Differential Equations
}

\author{
Khaled A. Gepreel, ${ }^{1,2}$ Taher A. Nofal, ${ }^{1,3}$ and Fawziah M. Alotaibi ${ }^{1}$ \\ ${ }^{1}$ Mathematics Department, Faculty of Science, Taif University, Taif, Saudi Arabia \\ ${ }^{2}$ Mathematics Department, Faculty of Science, Zagazig University, Zagazig, Egypt \\ ${ }^{3}$ Mathematics Department, Faculty of Science, El-Minia University, El-Minia, Egypt
}

Correspondence should be addressed to Khaled A. Gepreel; kagepreel@yahoo.com

Received 27 June 2013; Accepted 24 September 2013

Academic Editor: Mehmet Sezer

Copyright (C) 2013 Khaled A. Gepreel et al. This is an open access article distributed under the Creative Commons Attribution License, which permits unrestricted use, distribution, and reproduction in any medium, provided the original work is properly cited.

\begin{abstract}
We implement relatively analytical techniques, the homotopy perturbation method, and variational iteration method to find the approximate solutions for time and space fractional Benjamin-Bona Mahony equation. The fractional derivatives are described in the Caputo sense. These methods are used in applied mathematics to obtain the analytic approximate solutions for the nonlinear Bejamin-Bona Mahoney (BBM) partial fractional differential equation. We compare between the approximate solutions obtained by these methods. Also, we present the figures to compare between the approximate solutions. Also, we use the fractional complex transformation to convert nonlinear partial fractional differential equations to nonlinear ordinary differential equations. We use the improved $\left(G^{\prime} / G\right)$-expansion function method to find exact solutions of nonlinear fractional BBM equation.
\end{abstract}

\section{Introduction}

In recent years, there has been a great deal of interest in fractional differential equations. First there were almost no practical applications of fractional calculus, and it was considered by many as an abstract area containing only mathematical manipulations of little or no use. Nearly 30 years ago, the paradigm began to shift from pure mathematical formulations to applications in various fields. During the last decade, fractional calculus has been applied to almost every field of science, engineering, and mathematics. Several fields of application of fractional differentiation and fractional integration are already well established, and some others have just started. Many applications of fractional calculus can be found in turbulence and fluid dynamics, stochastic dynamical system, plasma physics and controlled thermonuclear fusion, nonlinear control theory, image processing, nonlinear biological systems, and astrophysics [1-11]. There has been some attempt to solve linear problems with multiple fractional derivatives (the so-called multiterm equations) $[1,12]$. Not much work has been done for nonlinear problems, and only a few numerical schemes have been proposed to solve nonlinear fractional differential equations. More recently, applications have included classes of nonlinear equation with multiorder fractional derivative, and this motivates us to develop a numerical scheme for their solutions [13]. Numerical and analytical methods have included Adomian decomposition method (ADM) [14-17], variational iteration method (VIM) [18-20], homotopy perturbation method [2124], homotopy analysis method [25-27], and the fractional complex transformation $[28,29]$ to get some special exact solutions for nonlinear partial fractional differential equation.

Recently, Zhao [30] investigated the nature of protein dynamics and thermodynamics. Olszewski [31] studied the uncertainty relation between intervals of energy and time derived for the electromagnetic radiation of a harmonic oscillator. El-Naggar et al. [32] investigated the initial stress, magnetic field, voids, and rotation effects on plane waves in generalized thermoelasticity. Khrennikov [33] discussed "Einstein's Dream" Quantum Mechanics as Theory of Classical Random Fields.

The main objective of this paper is to use two different methods such as homotopy perturbation method and 
variational iteration method for calculating the analytic approximate solutions of the Bejamin-Bona Mahoney equation. We compare between the approximate solutions which obtained from these methods. We make the figures to compare the approximate solutions. Also, we find some exact solutions to time and space fractional Bejamin-Bona Mahoney equation by using the improved $\left(G^{\prime} / G\right)$-expansion function method.

\section{Preliminaries and Notations}

We give some basic definitions and properties of the fractional calculus theory which are used further in this paper $[1,2]$. For the finite derivative $[a, b]$, we define the following fractional integral and derivatives.

Definition 1. If $f(t) \in L_{1}(a, b)$, the set of all integrable functions, and $\alpha>0$ then, the Riemann-Liouville fractional integral of order $\alpha$, denoted by $I_{a^{+}}^{\alpha}$, is defined by

$$
I_{a+}^{\alpha} f(\tau)=\frac{1}{\Gamma(\alpha)} \int_{a}^{t}(t-\tau)^{\alpha-1} f(\tau) d \tau .
$$

Definition 2. For $\alpha>0$, the Caputo fractional derivative of order $\alpha$, denoted by ${ }^{c} D_{a+}^{\alpha}$, is defined by

$$
{ }^{c} D_{a+}^{\alpha} f(t)=\frac{1}{\Gamma(n-\alpha)} \int_{a}^{t}(t-\tau)^{n-\alpha-1} D^{n} f(\tau) d \tau,
$$

where $n$ is such that $n-1<\alpha<n$ and $D=d / d \tau$.

If $\alpha$ is an integer, then this derivative takes the ordinary derivative

$$
{ }^{c} D_{a+}^{\alpha}=D^{\alpha}, \quad \alpha=1,2,3, \ldots
$$

Finally the Caputo fractional derivative on the whole space $R$ is defined by the following.

Definition 3. For $\alpha>0$, the Caputo fractional derivative of order $\alpha$ on the whole space, denoted by ${ }^{c} D_{+}^{\alpha}$, is defined by

$$
{ }^{c} D_{+}^{\alpha} f(x)=\frac{1}{\Gamma(n-\alpha)} \int_{-\infty}^{x}(x-\xi)^{n-\alpha-1} D^{n} f(\xi) d \xi .
$$

In recent years, in order to investigate the local behavior of fractional models, several local versions of fractional derivatives have been proposed, that is, the Kolwankar-Gangal local fractional derivative [35], Chen's fractal derivative [36], Cresson's derivative [37], and Jumarie's modified Riemann-Liouville derivative [38, 39]. Jumarie's derivative is defined as

$$
\begin{array}{r}
D_{x}^{\alpha} f(x)=\frac{1}{\Gamma(1-\alpha)} \frac{d}{d x} \times \int_{-\infty}^{x}(x-\xi)^{-\alpha}(f(\xi)-f(0)) d \xi, \\
0<\alpha<1,
\end{array}
$$

where $f: R \rightarrow R, x \rightarrow f(x)$ denotes a continuous (but not necessarily first-order-differentiable) function. We can obtain the following properties.
Property 1. Let $f(x)$ satisfy the definition of the modified Riemann-Liouville derivative, and let $f(x)$ be a $k$-order differentiable function. The generalized Taylor series is given by $[38,40]$

$$
f(x+h)=\sum_{k=0}^{\infty} \frac{h^{\alpha k}}{(\alpha k) !} f^{(\alpha k)}(x), \quad 0<\alpha<1 .
$$

Property 2. Assume that $f(x)$ denotes a continuous $R \rightarrow R$ function. We use the following equality for the integral with respect to $(d x)^{\alpha}[39,40]$ :

$$
I_{x}^{\alpha} f(x)=\frac{1}{\Gamma(\alpha)} \int_{0}^{x}(x-\xi)^{\alpha-1} f(\xi) d \xi .
$$

Property 3. Some useful formulas include

$$
\begin{gathered}
f^{(\alpha)}[x(t)] \frac{d f}{d x} x^{(\alpha)}(t), \\
D_{x}^{\alpha} x^{\beta}=\frac{\Gamma(1+\beta)}{\Gamma(1+\beta-\alpha)} x^{\beta-\alpha}, \\
\int(d x)^{\beta}=x^{\beta} .
\end{gathered}
$$

Function $f(x)$ should be differentiable with respect to $x(t)$, and $x(t)$ is fractional differentiable in (8). The above results are employed in the following sections. The modified Riemann-Liouville derivative has been successfully applied in the probability calculus [41], the fractional Laplace problems [42], the fractional variational approach with several variables [43], the fractional variational iteration method [44], the fractional variational approach with natural boundary conditions [45], and the fractional Lie group method [46].

\section{Basic Idea of the Homotopy Perturbation Method (HPM)}

In this section, we illustrate some basic concepts of the homotopy perturbation method for the following nonlinear partial fractional differential equation [21-24]:

$$
\begin{gathered}
D_{t}^{\alpha} u(\bar{x}, t)=v(\bar{x}, t)-\ell u(\bar{x}, t)-\check{N} u(\bar{x}, t), \\
m-1<\alpha<m, \quad m \in N, \quad t \geq 0, \quad \bar{x} \in R^{n},
\end{gathered}
$$

subject to the initial and boundary conditions

$$
\begin{array}{r}
u^{(i)}(0,0)=c_{i}, \quad B\left(u, \frac{\partial u}{\partial t}, \frac{\partial u}{\partial x_{j}}\right)=0, \\
i=0,1, \ldots, m-1, j=0,1, \ldots, n,
\end{array}
$$

where $\ell$ is a linear operator, while $\check{N}$ is a nonlinear operator, $v$ is a known analytical function, and $D_{t}^{\alpha}$ denotes the fractional derivative in the Caputo sense. The solution $u$ is assumed to be a causal function of time, that is, vanishing for $t<0$. Also, $u^{(i)}(\bar{x}, t)$ is the $i$ th derivative of $u, c_{i}, i=0,1, \ldots, m-1$, are the specified initial conditions, and $B$ is a boundary operator. 
Using He's homotopy perturbation technique [47], we can construct the following simple homotopy:

$$
\begin{array}{r}
(1-p) D_{t}^{\alpha} u(\bar{x}, t)+p\left[D_{t}^{\alpha} u(\bar{x}, t)+\ell u(\bar{x}, t)+\check{N} u(\bar{x}, t)\right. \\
-v(\bar{x}, t)]=0, \quad p \in[0,1],
\end{array}
$$

or

$$
\begin{array}{r}
D_{t}^{\alpha} u(\bar{x}, t)+p[\ell u(\bar{x}, t)+\check{N} u(\bar{x}, t)-v(\bar{x}, t)]=0, \\
p \in[0,1] .
\end{array}
$$

The homotopy parameter $p$ always changes from zero to unity. In the case of $p=0,(13)$ or (14) becomes

$$
D_{t}^{\alpha} u(\bar{x}, t)=0,
$$

and when $p=1,(13)$ or (14) turns out to be the original fractional differential equation.

Applying the homotopy perturbation method, we use the homotopy parameter $p$ to expand the solution into the following form:

$$
\begin{aligned}
u(\bar{x}, t)= & u_{0}(\bar{x}, t)+p u_{1}(\bar{x}, t)+p^{2} u_{2}(\bar{x}, t) \\
& +p^{3} u_{3}(\bar{x}, t)+\cdots .
\end{aligned}
$$

For nonlinear problems, let us set $\check{N} u(\bar{x}, t)=S(\bar{x}, t)$. Substituting (16) into (13) or (14) and equating the terms with identical powers of $p$, we can obtain a series of equations of the form

$$
\begin{gathered}
p^{0}: D_{t}^{\alpha} u_{0}(\bar{x}, t)=0, \\
p^{1}: D_{t}^{\alpha} u_{1}(\bar{x}, t)=-\ell u_{0}(\bar{x}, t)-S_{0}\left(u_{0}(\bar{x}, t)\right)+v(\bar{x}, t), \\
p^{2}: D_{t}^{\alpha} u_{2}(\bar{x}, t)=-\ell u_{1}(\bar{x}, t)-S_{1}\left(u_{0}(\bar{x}, t), u_{1}(\bar{x}, t)\right), \\
p^{3}: D_{t}^{\alpha} u_{3}(\bar{x}, t) \\
=-\ell u_{2}(\bar{x}, t)-S_{2}\left(u_{0}(\bar{x}, t), u_{1}(\bar{x}, t), u_{2}(\bar{x}, t)\right),
\end{gathered}
$$

and so on, where the functions $S_{0}, S_{1}, S_{2}, \ldots$ satisfy the following equations:

$$
\begin{aligned}
& S\left(u_{0}(\bar{x}, t)+p u_{1}(\bar{x}, t)+p^{2} u_{2}(\bar{x}, t)+\cdots\right) \\
& =S_{0}\left(u_{0}(\bar{x}, t)\right)+p S_{1}\left(u_{0}(\bar{x}, t), u_{1}(\bar{x}, t)\right) \\
& \quad+p^{2} S_{2}\left(u_{0}(\bar{x}, t), u_{1}(\bar{x}, t), u_{2}(\bar{x}, t)\right)+\cdots .
\end{aligned}
$$

Applying the operator $I_{t}^{\alpha}$ on both sides of (17) and considering the initial and boundary conditions, the terms of the series solution can be given by

$$
\begin{gathered}
u_{0}(\bar{x}, t)=\sum_{i=0}^{n-1} \frac{c_{i} t^{i}}{i !}, \\
u_{1}(\bar{x}, t)=-I_{t}^{\alpha}\left[L u_{0}(\bar{x}, t)\right] \\
-I_{t}^{\alpha}\left[S_{0}\left(u_{0}(\bar{x}, t)\right)\right]+I_{t}^{\alpha}[v(\bar{x}, t)],
\end{gathered}
$$

$$
\begin{aligned}
& u_{j}(\bar{x}, t)=-I_{t}^{\alpha}\left[\dot{L} u_{j-1}(\bar{x}, t)\right] \\
&-I_{t}^{\alpha}\left[S_{j-1}\left(u_{0}(\bar{x}, t), u_{1}(\bar{x}, t), \ldots, u_{j-1}(\bar{x}, t)\right)\right] \\
& j=2,3, \ldots
\end{aligned}
$$

On setting $p=1$, we get an accurate approximate solution in the following form:

$$
u(\bar{x}, t)=\sum_{i=0}^{\infty} u_{i}(\bar{x}, t)
$$

\section{Basic Idea of Variational Iteration Method (VIM)}

The basic concepts of the variational iteration method can be expressed as follows. Consider the differential equation of the form

$$
\ell u(x, t)+\check{N} u(x, t)=f(x, t),
$$

where $\ell$ is a linear operator, $\Sigma$ is a nonlinear operator, and $f(x, t)$ is the inhomogeneous term. According to VIM, a correction functional for (23) can be constructed as follows:

$$
\begin{array}{r}
u_{n+1}(x, t)=u_{n}(x, t) \\
+\int_{0}^{t} \lambda(s)\left(\ell u_{n}(x, s)+\check{N} \widetilde{u}_{n}(x, s)\right. \\
-f(x, s)) d s, \\
n=0,1,2, \ldots,
\end{array}
$$

where $\lambda$ is a general Lagrange multiplier, which can be identified optimally via the variational theory, the subscript $n$ denotes the $n$th approximations, and $\widetilde{u}_{n}$ is considered as restricted variation; that is, $\delta \widetilde{u}_{n}=0$. The successive approximations $u_{n+1}(x, t), n=0,1,2, \ldots$, of the solution $u(x, t)$ can be obtained after finding the Lagrange multiplier and by using the selective function $u_{0}(x, t)$ which is usually selected from initial conditions. Consequently, the solution is obtained as

$$
u(x, t)=\lim _{n \rightarrow \infty} u_{n}(x, t) .
$$

\section{Approximate Solutions for the Time and Space Fractional BBM Equation}

In this section, we use the homotopy perturbation method and variational iteration method to calculate the approximate solutions for the time and space fractional Bejamin-Bona Mahoney equation. Also, we compare the approximate solutions obtained from these methods.

5.1. The HPM for the Time and Space Fractional Nonlinear $B B M$ Equation. In this subsection, we use the HPM to 
construct the approximate solution of the time and space fractional nonlinear BBM equation in the form

$$
\begin{array}{r}
\frac{\partial^{\alpha} u}{\partial t^{\alpha}}+C \frac{\partial^{\beta} u}{\partial x^{\beta}}+L u^{2} \frac{\partial^{\beta} u}{\partial x^{\beta}}+N \frac{\partial^{\alpha}}{\partial t^{\alpha}}\left(\frac{\partial^{\beta}}{\partial x^{\beta}}\left(\frac{\partial^{\beta} u}{\partial x^{\beta}}\right)\right)=0 \\
t>0, \quad 0<\propto, \beta \leq 1,
\end{array}
$$

where $C, L$, and $N$ are constants.

With the initial condition,

$$
u(x, 0)=e^{-k x}
$$

By the homotopy perturbation technique, we construct a homotopy $V(r, p): \Omega \times[0,1] \rightarrow R$ which satisfies

$$
\begin{aligned}
H(V, p)=(1-p)\left[D_{t}^{\alpha} V-D_{t}^{\alpha} V_{0}\right] & \\
+ & p\left[D_{t}^{\alpha} V+C \frac{\partial^{\beta} V}{\partial x^{\beta}}+L V^{2} \frac{\partial^{\beta} V}{\partial x^{\beta}}\right. \\
& \left.+N \frac{\partial^{\alpha}}{\partial t^{\alpha}}\left(\frac{\partial^{\beta}}{\partial x^{\beta}}\left(\frac{\partial^{\beta} V}{\partial x^{\beta}}\right)\right)\right]=0, \quad r \in \Omega .
\end{aligned}
$$

According to the homotopy perturbation method, we can first use the embedding parameter $p$ as a small parameter and assume that the solution of (28) can be written as a power series in $p$ as follows:

$$
\begin{aligned}
V(x, t)= & V_{0}(x, t)+p V_{1}(x, t)+p^{2} V_{2}(x, t) \\
& +p^{3} V_{3}(x, t)+\cdots .
\end{aligned}
$$

Substituting (29) into (28) and arranging the coefficients of the powers of $p$, after some calculation we obtain

$$
\begin{gathered}
\frac{\partial^{\alpha} V_{0}}{\partial t^{\alpha}}=0 \\
\frac{\partial^{\alpha} V_{1}}{\partial t^{\alpha}}+C \frac{\partial^{\beta} V_{0}}{\partial x^{\beta}}+L V_{0}^{2} \frac{\partial^{\beta} V_{0}}{\partial x^{\beta}} \\
+N \frac{\partial^{\alpha}}{\partial t^{\alpha}}\left(\frac{\partial^{\beta}}{\partial x^{\beta}}\left(\frac{\partial^{\beta} V_{0}}{\partial x^{\beta}}\right)\right)=0 \\
\frac{\partial^{\alpha} V_{2}}{\partial t^{\alpha}}+C \frac{\partial^{\beta} V_{1}}{\partial x^{\beta}}+L V_{0}^{2} \frac{\partial^{\beta} V_{1}}{\partial x^{\beta}}+2 L V_{0} V_{1} \frac{\partial^{\beta} V_{0}}{\partial x^{\beta}} \\
+N \frac{\partial^{\alpha}}{\partial t^{\alpha}}\left(\frac{\partial^{\beta}}{\partial x^{\beta}}\left(\frac{\partial^{\beta} V_{1}}{\partial x^{\beta}}\right)\right)=0 \\
\frac{\partial^{\alpha} V_{3}}{\partial t^{\alpha}}+C \frac{\partial^{\beta} V_{2}}{\partial x^{\beta}}+L V_{0}^{2} \frac{\partial^{\beta} V_{2}}{\partial x^{\beta}} \\
+2 L V_{0} V_{1} \frac{\partial^{\beta} V_{1}}{\partial x^{\beta}}+2 L V_{0} V_{2} \frac{\partial^{\beta} V_{0}}{\partial x^{\beta}} \\
+L V_{1}^{2} \frac{\partial^{\beta} V_{0}}{\partial x^{\beta}}+N \frac{\partial^{\alpha}}{\partial t^{\alpha}}\left(\frac{\partial^{\beta}}{\partial x^{\beta}}\left(\frac{\partial^{\beta} V_{2}}{\partial x^{\beta}}\right)\right)=0
\end{gathered}
$$

and so on. We substitute the initial condition (27) into the system (30), then the approximate solutions of (26) take the following form:

$$
V_{0}(x, t)=e^{-k x}
$$

$$
V_{1}(x, t)=\frac{-t^{\alpha}}{\Gamma(\alpha+1)}\left[C(-k)^{\beta} e^{-k x}+L(-k)^{\beta} e^{-3 k x}\right] \text {, }
$$$$
V_{2}(x, t)=\frac{t^{2 \alpha}}{\Gamma(2 \alpha+1)} f_{1}\left(C+L V_{0}^{2}\right)
$$

$$
+2 \frac{t^{2 \alpha}}{\Gamma(2 \alpha+1)} L e^{-2 k x}(-k)^{\beta} f+N \frac{t^{\alpha}}{\Gamma(\alpha+1)} f_{2},
$$

$V_{3}(x, t)$

$$
\begin{aligned}
=-\left(C+L e^{-2 k x}\right)[ & \left(C+L(-2 k)^{\beta} e^{-2 k x}\right) f_{2} \frac{t^{3 \alpha}}{\Gamma(3 \alpha+1)} \\
& +2 L e^{-2 k x}(-2 k)^{\beta}(-k)^{\beta} \frac{t^{3 \alpha}}{\Gamma(3 \alpha+1)} f_{1} \\
& \left.+N f_{3} \frac{t^{2 \alpha}}{\Gamma(2 \alpha+1)}\right]
\end{aligned}
$$

$$
\begin{aligned}
& -2 L e^{-k x}\left(\frac{t^{2 \alpha}}{\Gamma(2 \alpha+1)}\right)^{2} f f_{1} \\
& -2 L e^{-k x}\left[\left(C+L V_{0}^{2}\right) \frac{t^{3 \alpha}}{\Gamma(3 \alpha+1)} f_{1}+2 L e^{-2 k x}(-k)^{\beta}\right.
\end{aligned}
$$

$$
\begin{array}{r}
\left.\times \frac{t^{3 \alpha}}{\Gamma(3 \alpha+1)} f+N \frac{t^{2 \alpha}}{\Gamma(2 \alpha+1)} f_{2}\right](-k)^{\beta} e^{-k x} \\
-L\left(\frac{t^{2 \alpha}}{\Gamma(2 \alpha+1)}\right)^{2} f^{2}(-k)^{\beta} e^{-k x} \\
-N\left[\left(C+L(-2 k)^{\beta} e^{-2 k x}\right) f_{3} \frac{t^{2 \alpha}}{\Gamma(2 \alpha+1)}+2 L e^{-2 k x}\right. \\
\left.\times(-2 k)^{2 \beta}(-k)^{\beta} \frac{t^{2 \alpha}}{\Gamma(2 \alpha+1)} f_{2}+N f_{4} \frac{t^{\alpha}}{\Gamma(\alpha+1)}\right],
\end{array}
$$

and so on. Thus the approximate solution of (26) is given by

$$
\begin{aligned}
u(x, t)= & e^{-k x}-\frac{t^{\alpha}}{\Gamma(\alpha+1)}\left[-C(-k)^{\beta} e^{-k x}-L(-k)^{\beta} e^{-3 k x}\right] \\
& +\frac{t^{2 \alpha}}{\Gamma(2 \alpha+1)} f_{1}\left(C+L e^{-2 k x}\right)
\end{aligned}
$$




$$
\begin{aligned}
& +2 \frac{t^{2 \alpha}}{\Gamma(2 \alpha+1)} L e^{-2 k x}(-k)^{\beta} f+N \frac{t^{\alpha}}{\Gamma(\alpha+1)} f_{2} \\
& -\left(C+L e^{-2 k x}\right)\left[\left(C+L(-2 k)^{\beta} e^{-2 k x}\right)\right. \\
& \times f_{2} \frac{t^{3 \alpha}}{\Gamma(3 \alpha+1)}+2 L e^{-2 k x}(-2 k)^{\beta} \\
& \times(-k)^{\beta} \frac{t^{3 \alpha}}{\Gamma(3 \alpha+1)} f_{1} \\
& \left.+N f_{3} \frac{t^{2 \alpha}}{\Gamma(2 \alpha+1)}\right] \\
& -2 L e^{-k x}\left(\frac{t^{2 \alpha}}{\Gamma(2 \alpha+1)}\right)^{2} f f_{1} \\
& -2 L e^{-k x}\left[\left(C+L V_{0}^{2}\right) \frac{t^{3 \alpha}}{\Gamma(3 \alpha+1)} f_{1}+2 L e^{-2 k x}\right. \\
& \left.\times(-k)^{\beta} \frac{t^{3 \alpha}}{\Gamma(3 \alpha+1)} f+N \frac{t^{2 \alpha}}{\Gamma(2 \alpha+1)} f_{2}\right] \\
& \times(-k)^{\beta} e^{-k x}-L\left(\frac{t^{2 \alpha}}{\Gamma(2 \alpha+1)}\right)^{2} f^{2}(-k)^{\beta} e^{-k x} \\
& -N\left[\left(C+L(-2 k)^{2 \beta} e^{-2 k x}\right) f_{3} \frac{t^{2 \alpha}}{\Gamma(2 \alpha+1)}\right. \\
& +2 L e^{-2 k x}(-2 k)^{2 \beta}(-k)^{\beta} \frac{t^{2 \alpha}}{\Gamma(2 \alpha+1)} f_{2} \\
& \left.+N f_{4} \frac{t^{\alpha}}{\Gamma(\alpha+1)}\right]+\cdots
\end{aligned}
$$

where

$$
\begin{aligned}
& f=C(-k)^{\beta} e^{-k x}+L(-k)^{\beta} e^{-3 k x}, \\
& f_{1}=C(-k)^{2 \beta} e^{-k x}+L(-k)^{\beta}(-3 k)^{\beta} e^{-3 k x}, \\
& f_{2}=C(-k)^{3 \beta} e^{-k x}+L(-k)^{\beta}(-3 k)^{2 \beta} e^{-3 k x}, \\
& f_{3}=C(-k)^{4 \beta} e^{-k x}+L(-k)^{\beta}(-3 k)^{3 \beta} e^{-3 k x}, \\
& f_{4}=C(-k)^{5 \beta} e^{-k x}+L(-k)^{\beta}(-3 k)^{4 \beta} e^{-3 k x}
\end{aligned}
$$

5.2. The VIM for the Time-Space Fractional Nonlinear BBM Equation. In this subsection, we use the VIM to construct the approximate solution of the time and space fractional nonlinear BBM equation (26).
The correction functional for (26) can be approximately expressed as follows:

$$
\begin{aligned}
& u_{n+1}(x, t) \\
& =u_{n}(x, t)+\int_{0}^{t} \lambda(\tau) \\
& \times\left[\frac{\partial^{\alpha} u_{n}(x, \tau)}{\partial t^{\alpha}}+C \frac{\partial^{\beta} u_{n}(x, \tau)}{\partial x^{\beta}}\right. \\
& +L\left(u_{n}(x, \tau)\right)^{2} \frac{\partial^{\beta} u_{n}(x, \tau)}{\partial x^{\beta}} \\
& \left.\quad+N \frac{\partial^{\alpha}}{\partial t^{\alpha}}\left(\frac{\partial^{\beta}}{\partial x^{\beta}}\left(\frac{\partial^{\beta} u_{n}(x, \tau)}{\partial x^{\beta}}\right)\right)\right] d \tau,
\end{aligned}
$$

where $\lambda$ is a general Lagrange multiplier, $u_{n}(x, \tau)$ is considered as restricted variations, and $\delta u_{n}$ is considered as a restricted variation. Making the above correction functional stationary and noticing that $\delta u_{n}=0$, we obtain

$$
\begin{gathered}
\delta u_{n+1}(x, t)=\delta u_{n}(x, t)+\int_{0}^{t} \delta \lambda(\tau)\left[\frac{\partial^{\alpha} u_{n}(x, \tau)}{\partial t^{\alpha}}\right] d \tau, \\
\delta u_{n+1}(x, t)=\delta u_{n}(x, t)+\lambda(\tau) \delta u_{n}(x, \tau) \\
+\int_{0}^{t} \delta u_{n}(x, \tau) \lambda \grave{(\tau)} d \tau=0 .
\end{gathered}
$$

Therefore, it can be identified as $\lambda=-1$, and the following variational iteration formula can be obtained:

$$
\begin{aligned}
& u_{n+1}(x, t)= u_{n}(x, t) \\
&-\int_{0}^{t}\left[\frac{\partial^{\alpha} u_{n}}{\partial t^{\alpha}}+C \frac{\partial^{\beta} u_{n}}{\partial x^{\beta}}+L\left(u_{n}\right)^{2} \frac{\partial^{\beta} u_{n}}{\partial x^{\beta}}\right. \\
&\left.+N \frac{\partial^{\alpha}}{\partial t^{\alpha}}\left(\frac{\partial^{\beta}}{\partial x^{\beta}}\left(\frac{\partial^{\beta} u_{n}}{\partial x^{\beta}}\right)\right)\right] d \tau .
\end{aligned}
$$

By the iteration formula (36), we can obtain the other components as

$$
\begin{gathered}
u_{0}=e^{-k x}, \\
u_{1}=e^{-k x}-f t, \\
u_{2}=3 e^{-k x}-3 f t+\frac{t^{2-\alpha}}{(2-\alpha) \Gamma(2-\alpha)}\left[f+N f_{2}\right] \\
+\frac{t^{4}}{4} L f^{2} f_{1}-\frac{t^{3}}{3}\left[2 L e^{-k x} f f_{1}+L f^{2}(-k)^{\beta} e^{-k x}\right] \\
+\frac{t^{2}}{2}\left[C f_{1}+L e^{-2 k x} f_{1}+2 L e^{-2 k x}(-k)^{\beta} f\right],
\end{gathered}
$$


TABLE 1: This table shows the absolute error between the approximate solutions (32), (38) and the approximate solution in [34] when $t=0.01$ and $0<x<1$.

\begin{tabular}{lccccc}
\hline$x$ & $u_{\mathrm{HPM}}$ obtained by [34] & abs $\left(u_{\mathrm{FHPM}}\right)$ & abs $\left(u_{\mathrm{FVIM}}\right)$ & $\left|u_{\mathrm{HPM}}-u_{\mathrm{FHPM}}\right|$ & $8.2 \times 10^{-5}$ \\
\hline 0.1 & 0.9212939744 & 0.9213768401 & 0.8630626710 & $6.8 \times 10^{-5}$ & $1.32 \times 10^{-1}$ \\
0.2 & 0.8324061770 & 0.8324750384 & 0.7892687927 & $5.7 \times 10^{-5}$ & $4.31 \times 10^{-2}$ \\
0.3 & 0.7522920995 & 0.7523498752 & 0.7203427038 & $4.8 \times 10^{-5}$ & $3.19 \times 10^{-2}$ \\
0.4 & 0.6800351886 & 0.6800841082 & 0.6563771349 & $4.1 \times 10^{-5}$ & $2.36 \times 10^{-2}$ \\
0.5 & 0.6148272679 & 0.6148690447 & 0.5973128723 & $3.5 \times 10^{-5}$ & $1.75 \times 10^{-2}$ \\
0.6 & 0.5559527414 & 0.5559886997 & 0.5429899342 & $3.1 \times 10^{-5}$ & $9.59 \times 10^{-3}$ \\
0.7 & 0.5027757211 & 0.5028068923 & 0.4931844956 & $2.7 \times 10^{-5}$ & $7.09 \times 10^{-3}$ \\
0.8 & 0.4547294332 & 0.4547566267 & 0.4476353171 & $2.3 \times 10^{-5}$ & $5.24 \times 10^{-3}$ \\
0.9 & 0.4113074114 & 0.4113312679 & 0.4060624384 & $2.1 \times 10^{-5}$ & $3.87 \times 10^{-3}$ \\
1 & 0.3720561063 & 0.3720771372 & 0.3681802017 & & \\
\hline
\end{tabular}

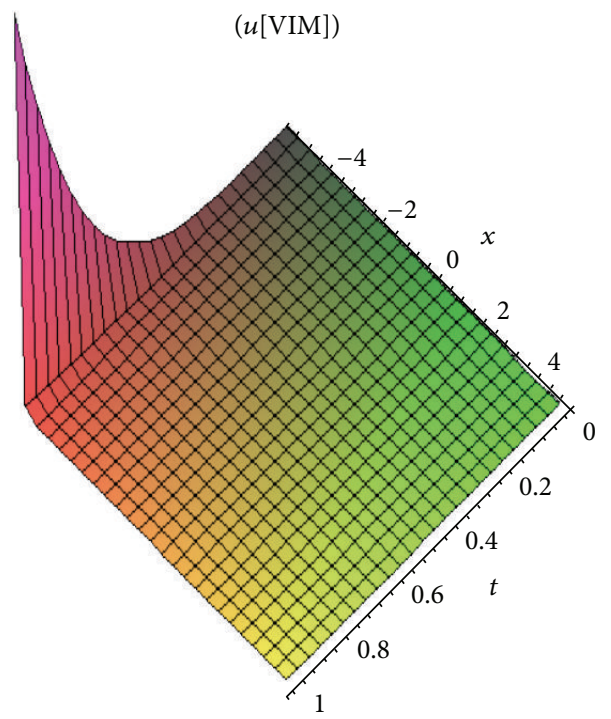

(a)

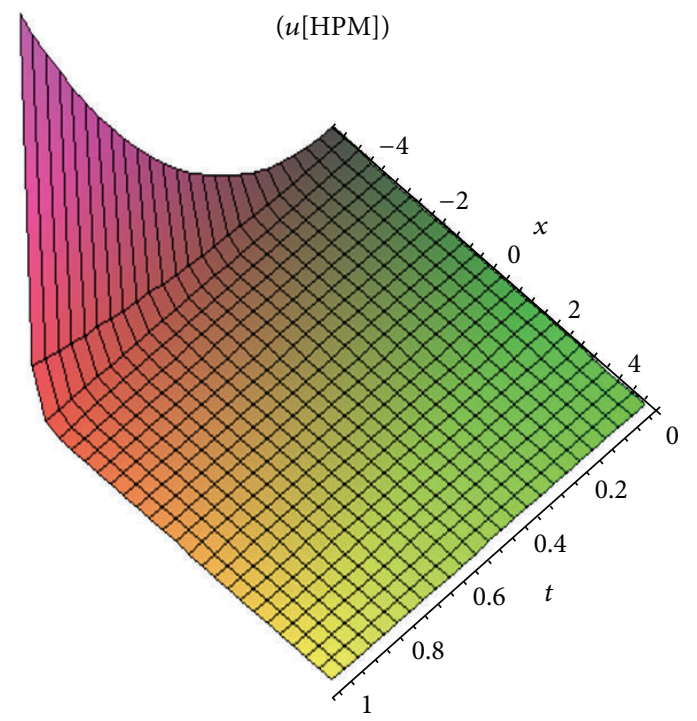

(b)

FIGURE 1: The approximate solution obtained by the variational iteration method (38) shown in (a) in comparison with that of the approximate solutions obtained by homotopy perturbation method (32) shown in (b) when $\alpha=1, \beta=1,-5<x<5$, and $0<t<1$.

and so on, and in the same manner, the rest of components of the iteration formula (36) can be obtained using Mathematica package where

$$
\begin{gathered}
f=C(-k)^{\beta} e^{-k x}+L(-k)^{\beta} e^{-3 k x}, \\
f_{1}=C(-k)^{2 \beta} e^{-k x}+L(-k)^{\beta}(-3 k)^{\beta} e^{-3 k x}, \\
f_{2}=C(-k)^{3 \beta} e^{-k x}+L(-k)^{\beta}(-3 k)^{2 \beta} e^{-3 k x} .
\end{gathered}
$$

The comparison between the approximate solutions which obtained, by using HPM (32) and VIM (38) is shown in Table 1.

Now we make the comparison between the approximate solutions obtained by the variation iteration method and the approximate solution obtained by the homotopy perturbation method as shown in Figures 1, 2, 3, 4, and 5.
Remark 4. From Figures 2-5, the approximate solution obtained by the homotopy perturbation method is different from the approximate solutions obtained by variational iteration when $\alpha$ and $\beta$ are not positive integers.

\section{The Improved $\left(G^{\prime} / G\right)$-Expansion Function Method}

Consider the following nonlinear partial fractional differential equation:

$$
\begin{aligned}
& F\left(u, D_{t}^{\alpha} u, D_{x}^{\beta} u, D_{y}^{\gamma} u, D_{z}^{\delta} u, D_{t}^{\alpha} D_{t}^{\alpha} u, D_{t}^{\alpha} D_{x}^{\beta} u,\right. \\
& \left.\quad D_{x}^{\beta} D_{x}^{\beta} u, D_{x}^{\beta} D_{y}^{\gamma} u, \ldots\right)=0, \quad 0<\alpha, \beta, \delta, \gamma<1,
\end{aligned}
$$




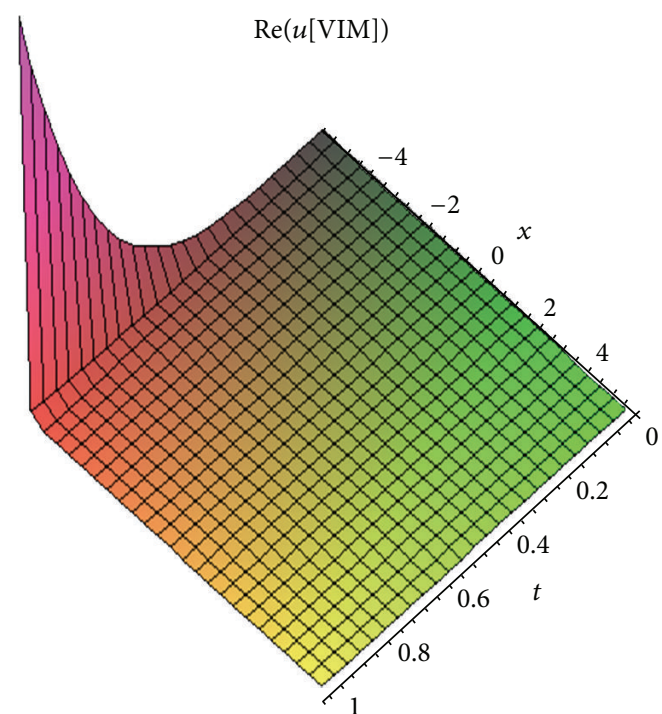

(a)

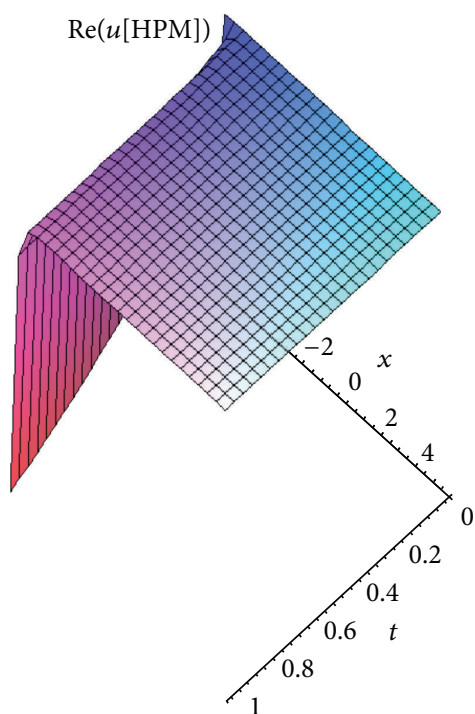

(b)

FIGURE 2: The real part of the approximate solution obtained by the variational itearation method (38) shown in (a) in comparison with the of that approximate solutions obtained by homotopy perturbation method (32) shown in (b) when $\alpha=0.2, \beta=0.4,-5<x<5$, and $0<t<1$.

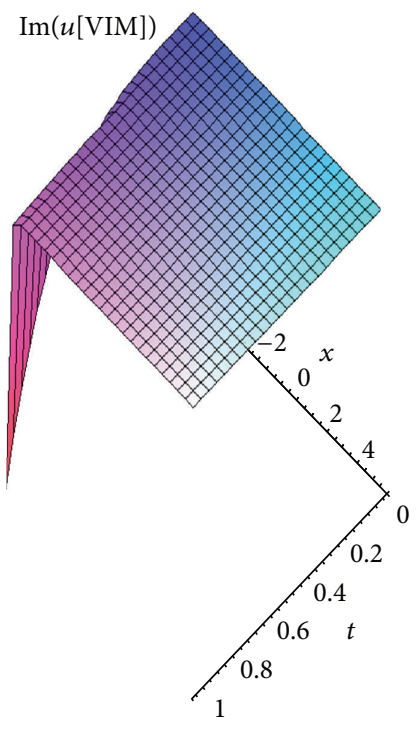

(a)

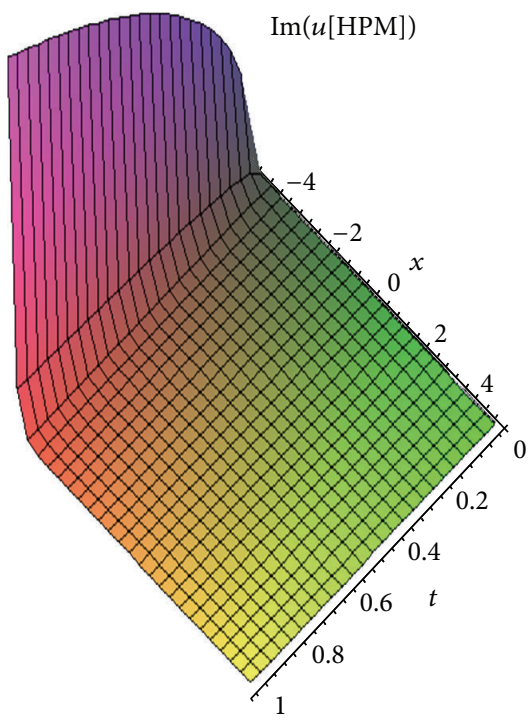

(b)

FIGURE 3: The imaginary part of the approximate solution obtained by the variational itearation method (38) shown in (a) in comparison with that of the approximate solutions obtained by homotopy perturbation method (32) shown in (b) when $\alpha=0.2, \beta=0.4,-5<x<5$, and $0<t<1$.

where $u$ is an unknown function and $F$ is a polynomial of $u$ and its partial fractional derivatives, in which the highest order derivatives and the nonlinear terms are involved.

In the following, we give the main steps of the improved $\left(G^{\prime} / G\right)$-expansion method.

Step 1. Li and He [48] proposed a fractional complex transformation to convert fractional differential equations into ordinary differential equations (ODE), so all analytical methods devoted to the advanced calculus can be easily applied to the fractional calculus. The traveling wave variable

$$
\begin{gathered}
u(x, y, z, t)=u(\xi), \\
\xi=\frac{R x^{\beta}}{\Gamma(\beta+1)}+\frac{Q y^{\gamma}}{\Gamma(\gamma+1)}+\frac{T z^{\delta}}{\Gamma(\delta+1)}+\frac{S t^{\alpha}}{\Gamma(\alpha+1)},
\end{gathered}
$$




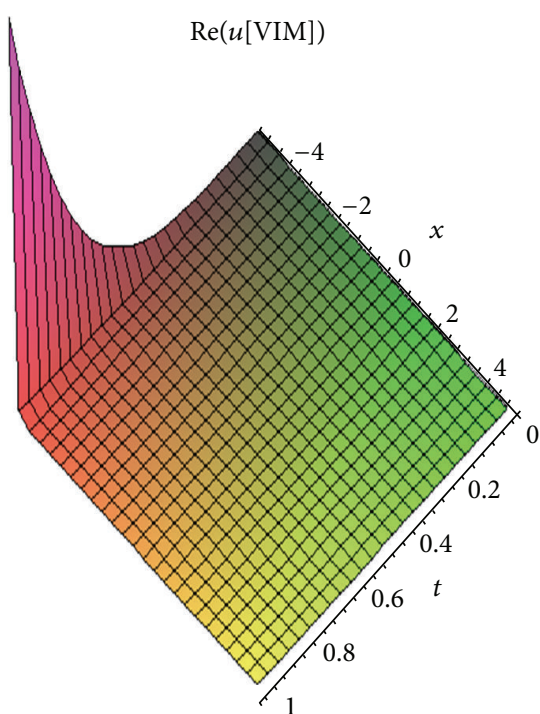

(a)

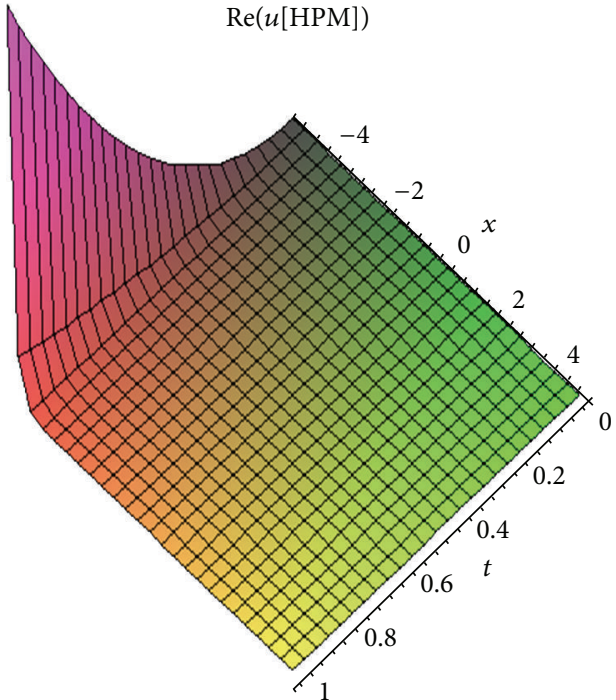

(b)

FIGURE 4: The real part of the approximate solution obtained by the variational itearation method (38) shown in (a) in comparison with that of the approximate solutions obtained by homotopy perturbation method (32) shown in (b) when $\alpha=0.99, \beta=0.99,-5<x<5$, and $0<t<1$.

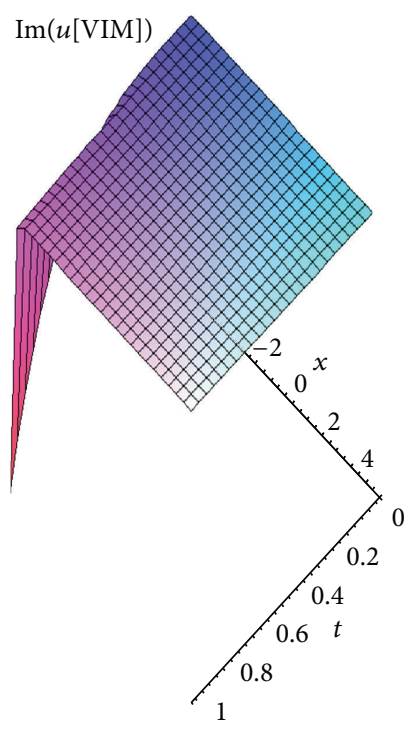

(a)

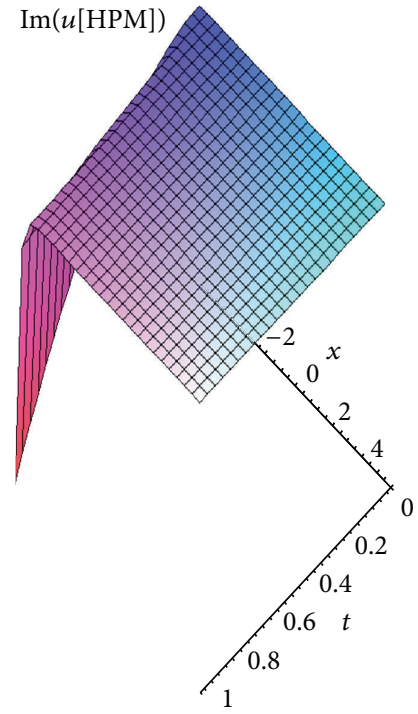

(b)

FIGURE 5: The imaginary part of the approximate solution which obtained by the variational itearation method (38) shown in (a) in comparison with the of the approximate solutions which obtained by homotopy perturbation method (32) shown in (b) when $\alpha=0.99, \beta=0.99$, $-5<x<5$, and $0<t<1$.

where $R, Q, T$, and $S$ are nonzero arbitrary constants, permits us to reduce (40) to an ODE of $u=u(\xi)$ in the form

$$
P\left(u, u^{\prime}, u^{\prime \prime}, u^{\prime \prime \prime}, \ldots\right)=0 .
$$

If possible, we should integrate (43) term by term one or more times.
Step 2. Suppose that the solution of (43) can be expressed as a polynomial of $\left(G^{\prime} / G\right)$ in the form [49-51]

$$
u(\xi)=\sum_{i=-m}^{m} a_{i}\left(\frac{G^{\prime}}{G}\right)^{i}, \quad a_{i} \neq 0,
$$


where $a_{i}(i=0, \pm 1, \ldots, \pm m)$ are constants, while $G(\xi)$ satisfies the following second-order linear ODE:

$$
G^{\prime \prime}(\xi)+\lambda G^{\prime}(\xi)+\mu G(\xi)=0,
$$

with $\lambda$ and $\mu$ being constants.

Step 3. The positive integer $m$ can be determined by considering the homogeneous balance between the highest order derivatives and the nonlinear terms appearing in (43). More precisely, we define the degree of $u(\xi)$ as $D[u(\xi)]=m$, which gives rise to the degrees of the other expressions as follows:

$$
\begin{gathered}
D\left[\frac{d^{q} u}{q^{q}}\right]=m+q, \\
D\left[u^{p}\left(\frac{d^{q} u}{q^{q}}\right)^{s}\right]=m p+s(q+m) .
\end{gathered}
$$

Therefore, we can obtain the value of $m$ in (44).

Step 4. Substituting (44) into (43), using (45), collecting all terms with the same order of $\left(G^{\prime} / G\right)$ together and equating each coefficient of the resulting polynomial to zero, we obtain a set of algebraic equations for $a_{i}(i=0, \pm 1, \ldots, \pm m), \lambda, \mu, R$, $Q, T$, and $S$.

Step 5. Since the general solutions to (45) are well known, then substituting $a_{i}(i=0, \pm 1, \ldots, \pm m), \lambda, \mu, R, Q, T, S$, and the general solutions of (45) into (44), we get more traveling wave solutions of the nonlinear partial fractional derivatives (40).

\section{Applications}

In this section, we use the improved $\left(G^{\prime} / G\right)$-expansion method to construct the exact solutions for some nonlinear partial fractional differential equations, via the time-space fractional nonlinear BBM equation.

We can see that the fractional complex transformation

$$
u(x, t)=U(\xi), \quad \xi=\frac{R x^{\beta}}{\Gamma(\beta+1)}+\frac{S t^{\alpha}}{\Gamma(\alpha+1)},
$$

where $R$ and $S$ are constants, permits us to reduce (26) to an ordinary differential equation. After that, we integrate to obtain

$$
(S+C R) U+\frac{L R}{3} U^{3}+N R^{2} S U^{\prime \prime}+c_{1}=0,
$$

where $c_{1}$ is the integration constant.

Considering the homogeneous balance between the highest order derivative and the nonlinear term in (48), we deduce that $n=1$. Thus, we get

$$
u(\xi)=a_{1}\left(\frac{G^{\prime}}{G}\right)+a_{0}+b_{1}\left(\frac{G^{\prime}}{G}\right)^{-1},
$$

where $a_{1}, a_{0}, b_{1}, R$, and $S$ are arbitrary constants to be determined later. Substituting (49) into (48), collecting all the terms of powers of $\left(G^{\prime} / G\right)$, and setting each coefficient to zero, we get a system of algebraic equations. With the aid of Maple or Mathematica, we can solve this system of algebraic equations to obtain the following sets of solutions.

Case 1

$$
\begin{gathered}
a_{0}=\frac{\lambda a_{1}}{2}, \quad S=-\frac{L a_{1}^{2}}{6 N R}, \\
\mu=\frac{12 C R^{2} N-2 L a_{1}^{2}+L a_{1}^{2} R^{2} \lambda^{2} N}{4 L a_{1}^{2} R^{2} N}, \\
b_{1}=c_{1}=0,
\end{gathered}
$$

where $\lambda, a_{1}, R, L, N$, and $C$ are arbitrary constants. Substituting (50) into (49) yields

$$
u(\xi)=\frac{\lambda a_{1}}{2}+a_{1}\left(\frac{G^{\prime}}{G}\right) .
$$

From the solutions of (45) and (51), we deduce the following types of traveling wave solutions for (26).

Family 1. If $\lambda^{2}-4 \mu>0$, then we have the hyperbolic solution

$$
\begin{aligned}
u(\xi)= & \frac{a_{1}}{2} \sqrt{\lambda^{2}-4 \mu} \\
& \times\left(\left(A \cosh \left((1 / 2) \sqrt{\left(\lambda^{2}-4 \mu\right) \xi}\right)\right.\right. \\
& \left.+B \sinh \left((1 / 2) \sqrt{\left(\lambda^{2}-4 \mu\right)} \xi\right)\right) \\
& \times\left(A \sinh \left((1 / 2) \sqrt{\left(\lambda^{2}-4 \mu\right)} \xi\right)\right. \\
& \left.\left.+B \cosh \left((1 / 2) \sqrt{\left(\lambda^{2}-4 \mu\right)} \xi\right)\right)^{-1}\right) .
\end{aligned}
$$

Family 2. If $\lambda^{2}-4 \mu<0$, then we have the trigonometric solution

$$
\begin{aligned}
& u(\xi)=\frac{a_{1}}{2} \sqrt{4 \mu-\lambda^{2}} \\
& \times\left(\left(-A \sin \left((1 / 2) \sqrt{\left(4 \mu-\lambda^{2}\right)} \xi\right)\right.\right. \\
&\left.+B \cos \left((1 / 2) \sqrt{\left(4 \mu-\lambda^{2}\right)} \xi\right)\right) \\
& \times\left(A \cos \left((1 / 2) \sqrt{\left(4 \mu-\lambda^{2}\right)} \xi\right)\right. \\
&\left.\left.+B \sin \left((1 / 2) \sqrt{\left(4 \mu-\lambda^{2}\right)} \xi\right)\right)^{-1}\right) .
\end{aligned}
$$

Family 3. If $\lambda^{2}-4 \mu=0$, then we have

$$
u(\xi)=\frac{a_{1} B}{A+B \xi}
$$


where

$$
\xi=\frac{R x^{\beta}}{\Gamma(\beta+1)}-\frac{L a_{1}^{2} t^{\alpha}}{6 N R \Gamma(\alpha+1)} .
$$

Case 2

$$
\begin{aligned}
& b_{1}=-\frac{6 C R^{2} N-L a_{1}^{2}}{8 L a_{1}^{2} R^{2} N}, \quad S=-\frac{L a_{1}^{2}}{6 N R}, \\
& \mu=\frac{6 C R^{2} N-L a_{1}^{2}}{8 L a_{1}^{2} R^{2} N}, \quad a_{0}=c_{1}=\lambda=0,
\end{aligned}
$$

where $a_{1}, R, L, N$, and $C$ are arbitrary constants. Substituting (56) into (49) yields

$$
u(\xi)=a_{1}\left(\frac{G^{\prime}}{G}\right)-\frac{6 C R^{2} N-L a_{1}^{2}}{8 L a_{1}^{2} R^{2} N}\left(\frac{G^{\prime}}{G}\right)^{-1} .
$$

From the solutions of (45) and (57), we deduce the following types of traveling wave solutions for (26).

Family 1. If $\mu>0$, then we have the hyperbolic solution

$$
\begin{gathered}
u(\xi)=a_{1}\left[\frac{1}{2} \sqrt{\mu}((A \cosh ((1 / 2) \sqrt{(\mu) \xi})\right. \\
+B \sinh ((1 / 2) \sqrt{(\mu) \xi})) \\
\times(A \sinh ((1 / 2) \sqrt{(\mu) \xi}) \\
\left.\left.+B \cosh ((1 / 2) \sqrt{(\mu) \xi}))^{-1}\right)\right] \\
-\frac{6 C R^{2} N-L a_{1}^{2}}{8 L a_{1}^{2} R^{2} N} \\
\times\left[\frac{1}{2} \sqrt{\mu}((A \cosh ((1 / 2) \sqrt{(\mu) \xi})\right. \\
+B \sinh ((1 / 2) \sqrt{(\mu) \xi})) \\
\times(A \sin h((1 / 2) \sqrt{(\mu) \xi}) \\
\left.\left.+B \cosh ((1 / 2) \sqrt{(\mu)} \xi))^{-1}\right)\right]^{-1}
\end{gathered}
$$

Family 2. If $\mu<0$, then we have the trigonometric solution

$$
\begin{gathered}
u(\xi)=a_{1}\left[\frac{1}{2} \sqrt{-\mu}((-A \sin ((1 / 2) \sqrt{(-\mu) \xi})\right. \\
+B \cos ((1 / 2) \sqrt{(-\mu) \xi})) \\
\times(A \cos ((1 / 2) \sqrt{(-\mu) \xi}) \\
-\frac{6 C R^{2} N-L a_{1}^{2}}{8 L a_{1}^{2} R^{2} N} \\
\times\left[\frac{1}{2} \sqrt{-\mu}\left((-A \sin ((1 / 2) \sqrt{(-\mu) \xi}))^{-1}\right)\right] \\
+B \cos ((1 / 2) \sqrt{(-\mu) \xi} \xi) \\
\times(A \cos ((1 / 2) \sqrt{(-\mu) \xi}) \\
\left.\left.+B \sin ((1 / 2) \sqrt{(-\mu) \xi}))^{-1}\right)\right]^{-1}
\end{gathered}
$$

Family 3. If $\mu=0$, then we have

$$
u(\xi)=a_{1}\left[\frac{B}{A+B \xi}\right]-\frac{6 C R^{2} N-L a_{1}^{2}}{8 L a_{1}^{2} R^{2} N}\left[\frac{B}{A+B \xi}\right]^{-1},
$$

where

$$
\xi=\frac{R x^{\beta}}{\Gamma(\beta+1)}-\frac{L a_{1}^{2} t^{\alpha}}{6 N R \Gamma(\alpha+1)} .
$$

\section{Conclusion}

In this paper, we use the homotopy perturbation method and variational iteration method to obtain the analytic approximate solutions for linear and nonlinear partial fractional differential equations. Section 5 shows the comparative study between the factional variational itearation methods and the homotopy perturbation method. The two methods are powerful and effective tools for the solutions of fractional partial differential equations, and both give two different approximate solutions of higher accuracy and closed form solutions if existing. Also, we use the improved $\left(G^{\prime} / G\right)$-expansion function method to calculate the exact solutions for the time and space fractional nonlinear BBM equation. This method is reliable and simple and gives many new exact solutions for the nonlinear fractional differential equations. When the parameters take certain special values, the solitary waves are derived from the traveling waves. This method is very efficient and powerful in finding the exact solutions for the nonlinear fractional differential equations. 


\section{References}

[1] I. Podlubny, Fractional Differential Equation, Academic Press, London, UK, 1999.

[2] S. G. Samko, A. A. Kilbas, and O. I. Marichev, Fractional Integrals and Derivatives: Theory and Applications, Gordon and Breach, Langhorne, Pennsylvania, 1993.

[3] A. A. Kilbas, H. M. Srivastava, and J. J. Trujillo, Theory and Applications of Fractional Differential Equations, vol. 204 of North-Holland Mathematical Studies, Elsevier, Amsterdam, The Netherlands, 2006.

[4] A. M. A. El-Sayed, "Fractional-order diffusion-wave equation," International Journal of Theoretical Physics, vol. 35, no. 2, pp. 311322, 1996.

[5] M. A. E. Herzallah, A. M. A. El-Sayed, and D. Baleanu, "On the fractional-order diffusion-wave process," Romanian Journal of Physics, vol. 55, no. 3-4, pp. 274-284, 2010.

[6] M. A. E. Herzallah, S. I. Muslih, D. Baleanu, and E. M. Rabei, "Hamilton-Jacobi and fractional like action with time scaling," Nonlinear Dynamics, vol. 66, no. 4, pp. 549-555, 2011.

[7] R. L. Magin, Fractional Calculus in Bioengineering, Begell House Publisher, West Redding, Conn, USA, 2006.

[8] B. J. West, M. Bologna, and P. Grigolini, Physics of Fractal Operators, Springer, New York, NY, USA, 2003.

[9] I. S. Jesus and J. A. Tenreiro MacHado, "Fractional control of heat diffusion systems," Nonlinear Dynamics, vol. 54, no. 3, pp. 263-282, 2008.

[10] O. P. Agrawal and D. Baleanu, "A Hamiltonian formulation and a direct numerical scheme for fractional optimal control problems," Journal of Vibration and Control, vol. 13, no. 9-10, pp. 1269-1281, 2007.

[11] V. E. Tarasov, "Fractional vector calculus and fractional Maxwell's equations," Annals of Physics, vol. 323, no. 11, pp. 27562778, 2008.

[12] J. H. He, "Some applications of nonlinear fractional differential equations and their approximations," Bulletin of Science, Technology and Society, vol. 15, no. 2, pp. 86-90, 1999.

[13] V. S. Erturk, S. Momani, and Z. Odibat, "Application of generalized differential transform method to multi-order fractional differential equations," Communications in Nonlinear Science and Numerical Simulation, vol. 13, no. 8, pp. 1642-1654, 2008.

[14] V. Daftardar-Gejji and S. Bhalekar, "Solving multi-term linear and non-linear diffusion-wave equations of fractional order by Adomian decomposition method," Applied Mathematics and Computation, vol. 202, no. 1, pp. 113-120, 2008.

[15] V. Daftardar-Gejji and H. Jafari, "Solving a multi-order fractional differential equation using Adomian decomposition," Applied Mathematics and Computation, vol. 189, no. 1, pp. 541548, 2007.

[16] E. M. E. Zayed, T. A. Nofal, and K. A. Gepreel, "Homotopy perturbation and Adomain decomposition methods for solving nonlinear Boussinesq equations," Communications on Applied Nonlinear Analysis, vol. 15, no. 3, pp. 57-70, 2008.

[17] M. A. E. Herzallah and K. A. Gepreel, "Approximate solution to the time-space fractional cubic nonlinear Schrodinger equation," Applied Mathematical Modelling, vol. 36, no. 11, pp. 56785685, 2012.

[18] N. H. Sweilam, M. M. Khader, and R. F. Al-Bar, "Numerical studies for a multi-order fractional differential equation," Physics Letters A, vol. 371, no. 1-2, pp. 26-33, 2007.
[19] J. H. He, "Preliminary report on the energy balance for nonlinear oscillations," International Journal of Non-Linear Mechanics, vol. 34, no. 4, pp. 699-708, 1999.

[20] G.-C. Wu and E. W. M. Lee, "Fractional variational iteration method and its application," Physics Letters A, vol. 374, no. 25, pp. 2506-2509, 2010.

[21] J.-H. He, "Homotopy perturbation method for solving boundary value problems," Physics Letters A, vol. 350, no. 1-2, pp. 8788, 2006.

[22] A. Golbabai and K. Sayevand, "Fractional calculus: a new approach to the analysis of generalized fourth-order diffusionwave equations," Computers \& Mathematics with Applications, vol. 61, no. 8, pp. 2227-2231, 2011.

[23] A. Golbabai and K. Sayevand, "The homotopy perturbation method for multi-order time fractional differential equations," Nonlinear Science Letters A, vol. 1, pp. 147-154, 2010.

[24] K. A. Gepreel, "The homotopy perturbation method applied to the nonlinear fractional Kolmogorov-Petrovskii-Piskunov equations," Applied Mathematics Letters, vol. 24, no. 8, pp. 1428-1434, 2011.

[25] K. A. Gepreel and M. S. Mohamed, "Analytical approximate solution for nonlinear space-time fractional Klein-Gordon equation," Chinese Physics B, vol. 22, no. 1, Article ID 010201, 2013.

[26] S. J. Liao, The Proposed Homotopy Analysis Technique for the Solution of Nonlinear Problem [Ph.D. thesis], Shanghai Jiao Tong University, 1992.

[27] S. J. Liao, "An approximate solution technique not depending on small parameters: a special example," International Journal of Non-Linear Mechanics, vol. 30, no. 3, pp. 371-380, 1995.

[28] J. H. He, "Asymptotic methods for solitary solutions and compactons," Abstract and Applied Analysis, vol. 2012, Article ID 916793, 130 pages, 2012.

[29] K. A. Gepreel and S. Omran, "Exact solutions for nonlinear partial fractional differential equations," Chinese Physics B, vol. 21, Article ID 110204, 2012.

[30] Q. Zhao, "Nature of protein dynamics and thermodynamics," Reviews in Theoretical Science, vol. 1, pp. 83-101, 2013.

[31] S. Olszewski, "A look on the scale of time useful in non-relativistic quantum mechanics," Quantum Matter, vol. 2, pp. 481-483, 2013.

[32] A. M. El-Naggar, Z. Kishka, A. M. Abd-Alla, I. A. Abbas, S. M. Abo-Dahab, and M. Elsagheer, "On the initial stress, magnetic field, voids and rotation effects on plane waves in generalized thermoelasticity," Journal of Computational and Theoretical Nanoscience, vol. 10, pp. 1408-1417, 2013.

[33] A. Khrennikov, “Einstein's Dream'-quantum mechanics as theory of classical random fields," Reviews in Theoretical Science, vol. 1, pp. 34-57, 2013.

[34] R. Asghari, "Application of the homotopy perturbation method to the modified BBM equation," Middle East Journal, vol. 10, no. 2, pp. 274-276, 2011.

[35] K. M. Kolwankar and A. D. Gangal, "Local fractional FokkerPlanck equation," Physical Review Letters, vol. 80, no. 2, pp. 214217, 1998.

[36] W. Chen and H. G. Sun, "Multiscale statistical model of fullydeveloped turbulence particle accelerations," Modern Physics Letters B, vol. 23, no. 3, article 449, 2009.

[37] J. Cresson, "Non-differentiable variational principles," Journal of Mathematical Analysis and Applications, vol. 307, no. 1, pp. 48-64, 2005. 
[38] G. Jumarie, "Modified Riemann-Liouville derivative and fractional Taylor series of nondifferentiable functions further results," Computers \& Mathematics with Applications, vol. 51, no. 9-10, pp. 1367-1376, 2006.

[39] G. Jumarie, "Lagrange characteristic method for solving a class of nonlinear partial differential equations of fractional order," Applied Mathematics Letters, vol. 19, no. 9, pp. 873-880, 2006.

[40] G.-C. Wu, "A fractional characteristic method for solving fractional partial differential equations," Applied Mathematics Letters, vol. 24, no. 7, pp. 1046-1050, 2011.

[41] G. Jumarie, "New stochastic fractional models for Malthusian growth, the Poissonian birth process and optimal management of populations," Mathematical and Computer Modelling, vol. 44, no. 3-4, pp. 231-254, 2006.

[42] G. Jumarie, "Laplace's transform of fractional order via the Mittag-Leffler function and modified Riemann-Liouville derivative," Applied Mathematics Letters, vol. 22, no. 11, pp. 1659-1664, 2009.

[43] R. Almeida, A. B. Malinowska, and D. F. M. Torres, "A fractional calculus of variations for multiple integrals with application to vibrating string," Journal of Mathematical Physics, vol. 51, no. 3, Article ID 033503, p. 12, 2010.

[44] K. A. Gepreel and A. A. Al-thobaiti, "Exact solutions of nonlinear partial fractional sub-equation method," Indian Journal of Physics, 2014.

[45] A. B. Malinowska, M. R. Sidi Ammi, and D. F. M. Torres, "Composition functionals in fractional calculus of variations," Communications in Fractional Calculus, vol. 1, no. 1, pp. 32-40, 2010.

[46] G.-C. Wu, "A fractional lie group method for anomalous diffusion equations," Communications in Fractional Calculus, vol. 1, pp. 27-31, 2010.

[47] J. H. He, "Some asymptotic methods for strongly nonlinear equations," International Journal of Modern Physics B, vol. 20, no. 10, pp. 1141-1199, 2006.

[48] Z.-B. Li and J.-H. He, "Fractional complex transform for fractional differential equations," Mathematical \& Computational Applications, vol. 15, no. 5, pp. 970-973, 2010.

[49] M. Wang, X. Li, and J. Zhang, "The $\left(G^{\prime} / G\right)$-expansion method and travelling wave solutions of nonlinear evolution equations in mathematical physics," Physics Letters A, vol. 372, no. 4, pp. 417-423, 2008.

[50] E. M. E. Zayed and K. A. Gepreel, "The $\left(G^{\prime} / G\right)$-expansion method for finding traveling wave solutions of nonlinear partial differential equations in mathematical physics," Journal of Mathematical Physics, vol. 50, no. 1, p. 013502, 12, 2009.

[51] H. Zhang, "New exact complex travelling wave solutions to nonlinear Schrödinger (NLS) equation," Communications in Nonlinear Science and Numerical Simulation, vol. 14, no. 3, pp. 668673, 2009. 


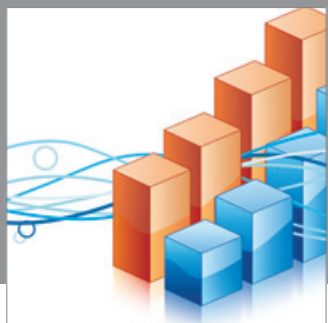

Advances in

Operations Research

mansans

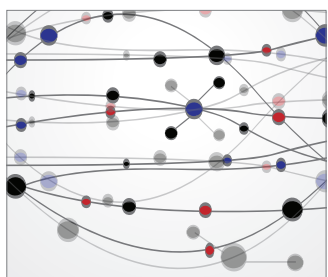

The Scientific World Journal
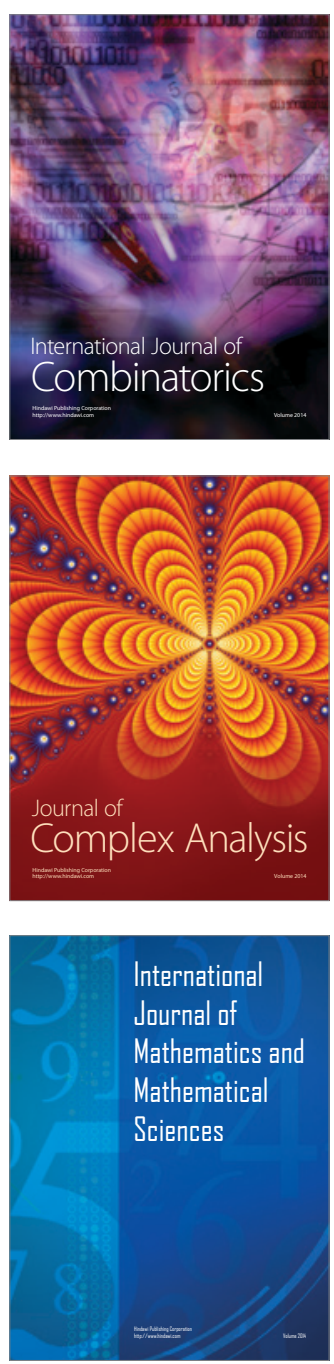
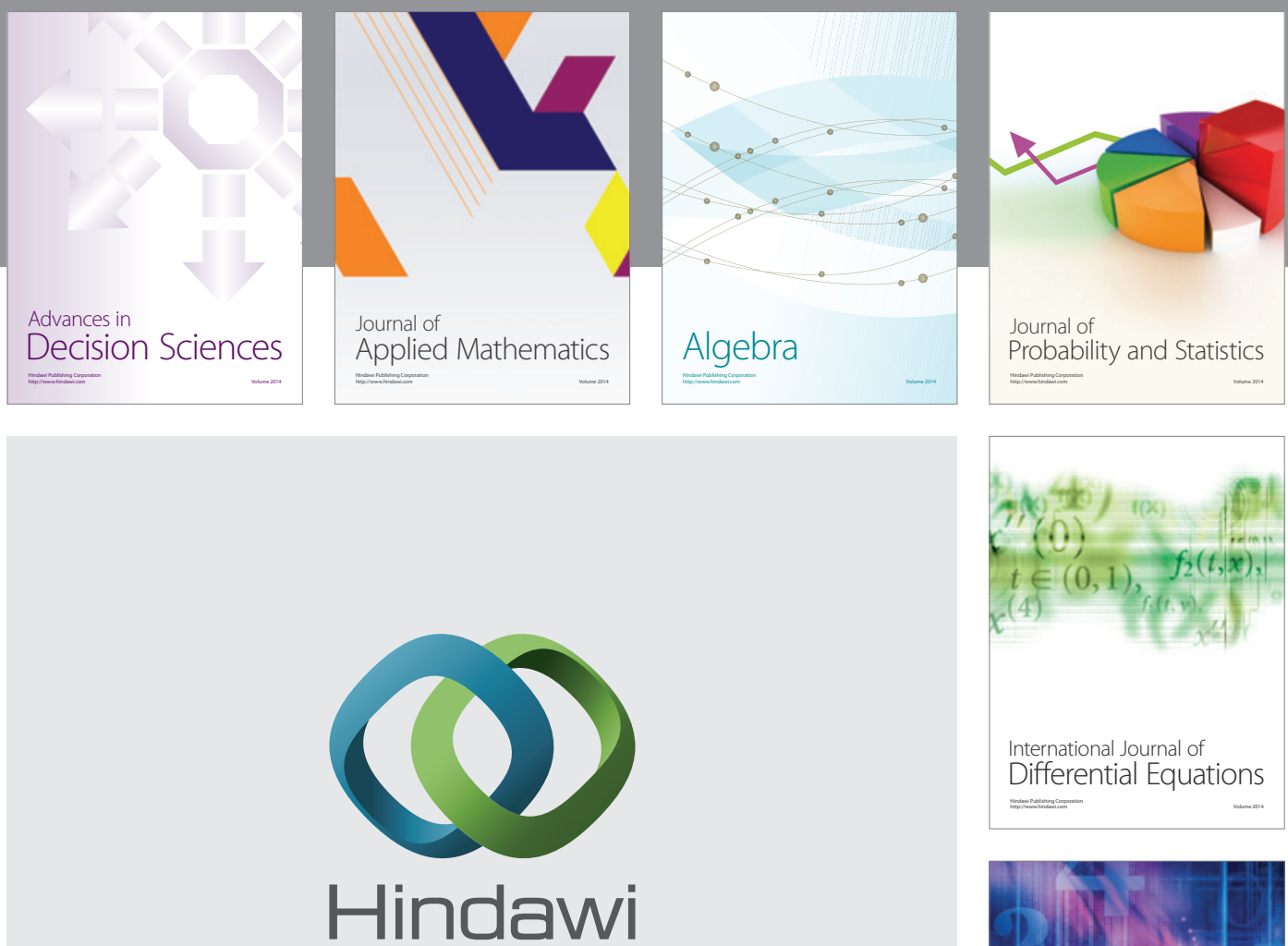

Submit your manuscripts at http://www.hindawi.com
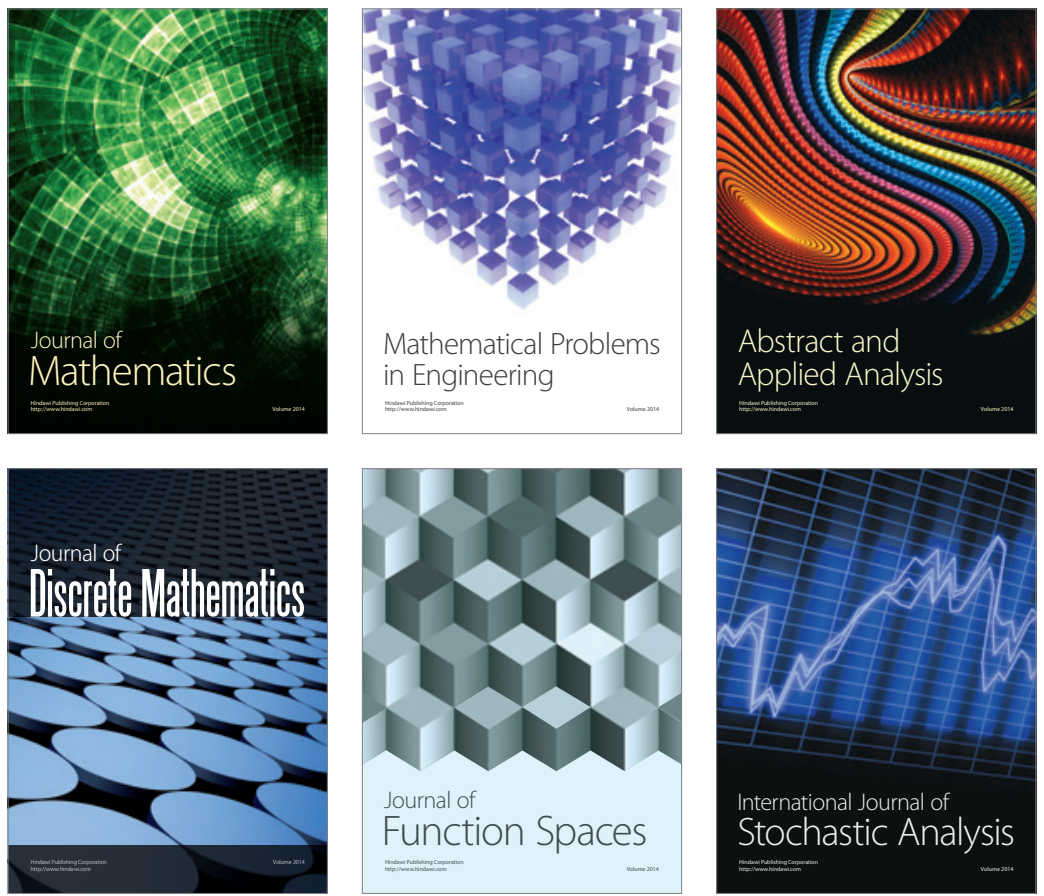

Journal of

Function Spaces

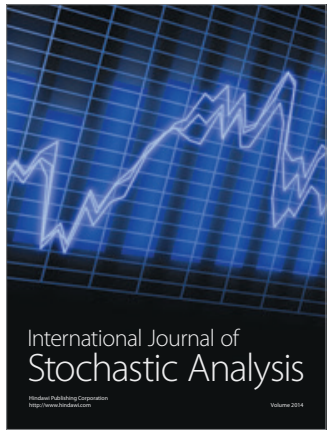

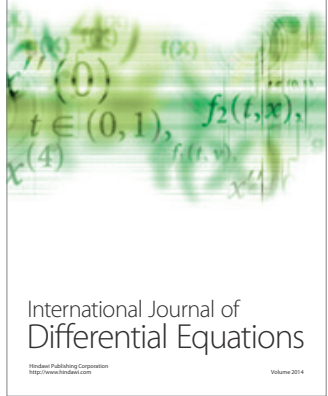
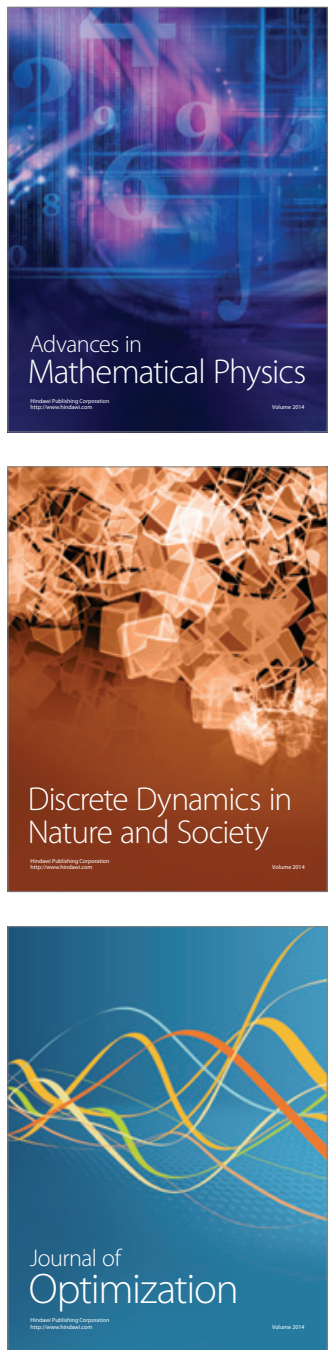